Reprod. Nutr. Develop., 1988, 28 (3 A), 563-572

\title{
Variations nycthémérales de l'incorporation de l'acide $\gamma$-aminobutyrique (GABA) et de la taurine tritiés par l'épiphyse de la souris CD
}

\author{
Anne-Lise DELEZOIDE, Véronique ALADJAJIAN, Chantal GUERILLOT, \\ Marie-Thérèse SIMON, A. PFISTER, C. DA LAGE \\ avec la collaboration technique de Léa BONNION
}

Laboratoire d'Histologie et Embryologie,

Faculté de Médecine Necker-Enfants Malades,

156, rue de Vaugirard. 75015 Paris.

Summary. Circadian variations in the uptake of two neurotransmitters, $\gamma$-aminobutyric acid (GABA) and taurine in $C D$ mice.

Pineal uptake of two putative neurotransmitters, $\gamma$-aminobutyric acid (GABA) and taurine, was investigated during the $24 \mathrm{~h}$-cycle in mice kept in long (14L/10D) or short (10L/14D) photoperiods. Tritiated mediators were intraperitoneally injected and their concentration both in pineal gland and cerebellum were measured at several times along the nycthemere. A circadian rhythm of exogenous GABA uptake was observed in mice kept in long photoperiods: GABA uptake was high during the night, when the gland was active and low during the day. It corresponded to the pineal cycle of indoleamine synthesis. It was particularly sensitive to sudden changes of lighting. In short photoperiods, a similar cycle was observed but the result was not significant because of individual variations.

Taurine uptake did not show such a circadian rhythm either in long or in short photoperiods: it was constant and rather low with large variations from one animal to another.

\section{Introduction.}

L'épiphyse synthétise des indolamines selon des rythmes circadiens qui dépendent étroitement des photopériodes. La noradrénaline (NA) a un effet essentiel sur l'activation nocturne de la $\mathrm{N}$-acétyltransférase (NAT) pinéalocytaire, effet qui est arrêté brutalement par la lumière (Axelrod et Zatz, 1977). La noradrénaline n'est certainement pas seule à intervenir, ni libre d'agir, sans divers contrôles locaux. Cette dernière idée ressort de la découverte de nombreux neuromédiateurs potentiels dans le tissu épiphysaire. Les dosages biochimiques, les méthodes chromatographiques et l'histochimie ont permis de trouver dans la pinéale divers transmetteurs à des concentrations notables. Nous nous sommes intéressés à l'activité de deux d'entre eux : l'acide $\gamma$-aminobutyrique et la taurine. 
Le $G A B A$, mis en évidence dans la pinéale par Labella et al. en 1968, puis par d'autres auteurs (Vellan et al., 1970; Mata et al., 1976) siège avant tout, chez le rat, dans les cellules gliales (Young et al., 1973). La glande est capable de le synthétiser (Kanazawa et al., 1976), de le capter et de le dégrader (Shon et al., 1975). Forchetti (1979) a montré que cet acide aminé pouvait inhiber l'excrétion de la noradrénaline, de la dopamine et de la sérotonine. Ebadi et Chan (1980) ont indiqué que le GABA exerce chez les bovins une régulation négative sur la synthèse de mélatonine, en inhibant l'activité enzymatique de la NAT. Ce rôle de médiateur ressort également des travaux de Waniewski et Suria (1977), qui décrivent chez le rat une variation circadienne du GABA épiphysaire endogène : ce rythme est inversé par rapport à celui de la noradrénaline.

La taurine, présente également en grande quantité dans la pinéale (Barbeau et al., 1968 ; Rosenblum et al., 1979), a une action sur la synthèse et l'excrétion de la mélatonine (Baskin et Dagirmanjian, 1973). Elle peut être captée à partir du plasma et surtout être synthétisée par la glande (Ebels et al., 1980). De plus, Grosso et al. (1978) ont démontré l'existence d'un rythme circadien de la taurine endogène dans l'épiphyse du rat. Divers travaux permettent de considérer la taurine comme antagoniste du GABA au niveau de la rétine et du cervelet (Namina et al., 1982; Adler, 1983).

Dans ce travail nous avons voulu préciser les éventuelles variations circadiennes de l'incorporation épiphysaire de GABA et de taurine tritiés chez des souris soumises à des photopériodes courtes ou longues; nous avons choisi de travailler sur la souris qui présente des variations fonctionnelles nycthémérales, comme l'ont montré Upson et al., en 1976 et Matsushima et al., en 1977.

\section{Matériel et méthodes.}

\section{Produits radioactifs.}

Le ${ }^{3} H-G A B A$ utilisé provient du Commissariat à l'Energie Atomique et a une activité spécifique de $50 \mathrm{Ci} / \mathrm{mM}$. Il est injecté par voie intrapéritonéale après dilution extemporanée dans une solution physiologique à raison de $50 \mu \mathrm{Ci} / 0,5 \mathrm{ml} /$ animal.

La ${ }^{3} \mathrm{H}$-taurine provient du Centre d'Amersham, UK, et a une activité spécifique de $12,8 \mathrm{Ci} / \mathrm{mM}$. Elle est utilisée par voie intrapéritonéale à raison de $25 \mu \mathrm{Ci} / 0,5 \mathrm{ml} /$ animal après dilution dans une solution physiologique $(\mathrm{NaCl}$ $0,9 \%)$.

Au cours d'expériences préliminaires (résultats non publiés), nous avons défini le temps de captage maximum du GABA et de la taurine par la pinéale de souris. Après injection intrapéritonéale d'une dose connue de GABA tritié $(50 \mu \mathrm{Ci}$ par animal) ou de taurine tritiée $(25 \mu \mathrm{Ci}$ par animal), deux souris par précurseur sont sacrifiées respectivement 5,10,15,20,30 et $60 \mathrm{~min}$ après l'injection. Les épiphyses sont immédiatement prélevées, la radioactivité de chacune d'elles est mesurée au compteur à scintillation. Le maximum d'incorporation est observé 
après $15 \mathrm{~min}$ pour le ${ }^{3} \mathrm{H}-\mathrm{GABA}$, avec $410 \mathrm{cpm}$ et $30 \mathrm{~min}$ pour la ${ }^{3} \mathrm{H}$-taurine, avec $816 \mathrm{cpm}$. Ces deux temps sont retenus pour la détermination du délai entre l'injection et le sacrifice.

\section{Protocole expérimental.}

Nous avons utilisé 144 souris mâles de souche CD pesant chacune environ $30 \mathrm{~g}$ au moment du sacrifice. Ces animaux proviennent d'un centre d'élevage (Charles River France) où les conditions d'éclairement sont de $12 \mathrm{~h}$ de lumière (L) et de $12 \mathrm{~h}$ d'obscurité (Ob) par $24 \mathrm{~h}$. Les animaux sont répartis en deux groupes égaux, soumis à des conditions photopériodiques opposées selon les modalités suivantes:

1) Photopériodes longues. -- Soixante-douze animaux sont acclimatés pendant 30 jours dans une animalerie climatisée $\left(22{ }^{\circ} \mathrm{C}\right.$ et $45 \%$ d'humidité $)$ où la lumière est allumée à $7 \mathrm{~h}$ et éteinte à $21 \mathrm{~h}$ (soit $14 \mathrm{~h}$ de lumière et $10 \mathrm{~h}$ d'obscurité). Ils sont nourris (aliments UAR) et abreuvés ad libitum. A la fin de cette période, ils sont sacrifiés par décapitation $15 \mathrm{~min}$ après injection de ${ }^{3} \mathrm{H}-\mathrm{GABA}$ ou $30 \mathrm{~min}$ après injection de ${ }^{3} \mathrm{H}$-taurine.

2) Photopériodes courtes. - Les 72 autres souris sont acclimatées dans les mêmes conditions de température, d'hygrométrie et d'alimentation mais avec un cycle d'éclairement comportant $10 \mathrm{~h}$ de lumière et $14 \mathrm{~h}$ d'obscurité (lumière allumée à $8 \mathrm{~h}$ et éteinte à $18 \mathrm{~h}$ ). Elles sont sacrifiées de la même façon que les précédentes après quatre semaines.

Dans chacun des deux groupes, les souris sont réparties en six lots dont les animaux sont sacrifiés à différents moments du nycthémère. Nous nous sommes particulièrement attachés à explorer les périodes de changement brutal du régime lumineux : Lot 1 : milieu de la période d'obscurité ; Lot 2: 30 min avant la fin de la période d'obscurité ; Lot 3:30 min après le début de la période lumineuse ; Lot 4 : milieu de la période lumineuse; Lot $5: 30$ min avant la fin de la période lumineuse ; Lot $6: 30$ min après le début de la période d'obscurité.

Les animaux sont pesés la veille du jour choisi pour le sacrifice. Celui-ci est réalisé par décapitation, après une brève anesthésie à l'éther. Pendant les périodes obscures les sacrifices sont réalisés sous un très faible éclairage en lumière rouge $(\lambda=600 \mathrm{~nm}$; intensité : 10 watts ; distance : $50 \mathrm{~cm})$.

\section{Prélèvements et mesures.}

Les épiphyses sont prélevées en deux à trois minutes sous une loupe binoculaire, ainsi qu'un petit fragment de vermis cérébelleux qui est pesé. Le poids de l'épiphyse est déterminé à partir des calculs de Legait et Oboussier (1977) : il correspond à $7,7 \times 10^{-6}$ du poids corporel; nous nous sommes résolus à cet expédient car l'épiphyse fraîche et non fixée est à la fois très petite et très fragile ; elle adhère aux objets et supporte difficilement les manipulations. Les tissus 
prélevés sont aussitôt dissous à $50^{\circ} \mathrm{C}$ dans quelques gouttes de Soluène (Packard) pendant $12 \mathrm{~h}$. Chaque échantillon reçoit alors $10 \mathrm{ml}$ de milieu de scintillation (Dimilume Packard) avant d'être réfrigéré et placé dans un compteur à scintillation Packard.

La concentration de la molécule marquée dans le cervelet ou l'épiphyse est calculée en divisant le nombre de désintégrations par minute mesuré dans l'échantillon par le nombre correspondant à la totalité du marqueur injecté, rapporté lui-même au poids du fragment; elle est exprimée en \% dans les tableaux 1 et 2 . Des comparaisons statistiques entre les lots de chaque groupe et entre les deux groupes sont effectuées en utilisant les tests $\mathrm{F}$ de Fisher (analyse de variance) et t de Student.

\section{Résultats.}

$A-G A B A$.

Au niveau du cervelet nous avons calculé l'accumulation du GABA tritié en mesurant la radioactivité par $\mathrm{mg}$ de cet organe par rapport à la répartition du marqueur supposée homogène dans tout l'organisme.

L'examen des résultats (tabl. 1) montre que le cervelet incorpore modérément et de matière homogène le GABA tritié. On n'observe pas de modifications circadiennes du captage, mais ce dernier est légèrement supérieur chez les animaux soumis à des photopériodes longues $(p<0,0001)$.

\section{TABLEAU 1}

Concentration moyenne, dans le cervelet de souris, du GABA tritié injecté par voie intrapéritonéale pour les douze animaux qui composent chaque lot. M est la moyenne obtenue sur l'ensemble des souris d'un groupe quand aucune différence significative n'apparaît statistiquement entre les six lots du groupe.

\section{Concentration}

moyenne du GABA

tritié par mg de

Lot 1

Lot 2

Lot 3

Lot 4

Lot 5

Lot 6

cervelet de

souris (\%)

\begin{tabular}{lllllll}
\hline $\begin{array}{l}\text { Photopériode } \\
\text { longue } \\
M=42 \pm 1\end{array}$ & $43 \pm 6$ & $41 \pm 12$ & $43 \pm 15$ & $42 \pm 12$ & $40 \pm 13$ & $41 \pm 6$ \\
\hline $\begin{array}{l}\text { Photopériode } \\
\text { courte } \\
M=32 \pm 5\end{array}$ & $34 \pm 8$ & $31 \pm 6$ & $32 \pm 3$ & $30 \pm 3$ & $34 \pm 7$ & $33 \pm 4$ \\
\hline
\end{tabular}


Au niveau de la pinéale l'étude de la quantité de radioactivité acquise par chaque épiphyse (cpm/épiphyse) et la concentration du produit marqué dans la glande apportent des résultats équivalents.

En photopériode longue on observe des différences statistiquement significatives ( $p<0,001$ ) entre les lots au cours du nycthémère (fig. $1 a)$. L'épiphyse de souris incorpore le GABA exogène suivant un rythme circadien, le captage étant plus important la nuit que le jour. L'avidité de la pinéale est assez faible pendant la phase lumineuse; elle augmente brutalement au début de la phase sombre et passe par un maximum au milieu de la nuit. Cette valeur élevée se maintient jusqu'à la fin de la période d'obscurité ; elle baisse ensuite progressivement pour atteindre un minimum au milieu du jour.

En photopériode courte les résultats obtenus ne permettent pas de décrire un rythme circadien d'incorporation du GABA. Bien que non significatives, les variations observées sont parallèles aux précédentes (fig. 1b). Les niveaux d'incorporation en phase nocturne sont statistiquement superposables dans les deux groupes. Il semble donc que l'avidité de l'épiphyse pour le GABA soit peu influencée par les conditions photopériodiques.

\section{$B-$ Taurine.}

Le cervelet ne capte qu'une quantité minime de cet acide aminé. Pour tous les lots étudiés les résultats sont faibles mais constants (tabl. 2).

TABLEAU 2

Concentration moyenne de la taurine tritiée dans le cervelet de souris.

\begin{tabular}{lcccccc}
\hline $\begin{array}{l}\text { Concentration } \\
\text { de la taurine tritiée } \\
\text { dans le cervelet } \\
\text { de souris (\%) }\end{array}$ & Lot 1 & Lot 2 & Lot 3 & Lot 4 & Lot 5 & Lot 6 \\
\hline $\begin{array}{l}\text { Photopériode } \\
\text { longue } \\
\text { (NS) }\end{array}$ & 4 & 5 & 5 & 5 & 5 & 5 \\
\hline $\begin{array}{l}\text { Photopériode } \\
\text { courte } \\
\text { (NS) }\end{array}$ & 4 & 5 & 4 & 6 & 4 & 4 \\
\hline
\end{tabular}

Dans l'épiphyse l'incorporation de taurine exogène est importante, mais elle ne subit pas de fluctuations circadiennes. Le captage est statistiquement stable autour des mêmes valeurs quelles que soient l'heure du prélèvement et la durée de la photopériode (fig. $2 a$ et $2 b$ ). On note cependant de grandes variations individuelles dans les réponses des souris de chaque lot. 


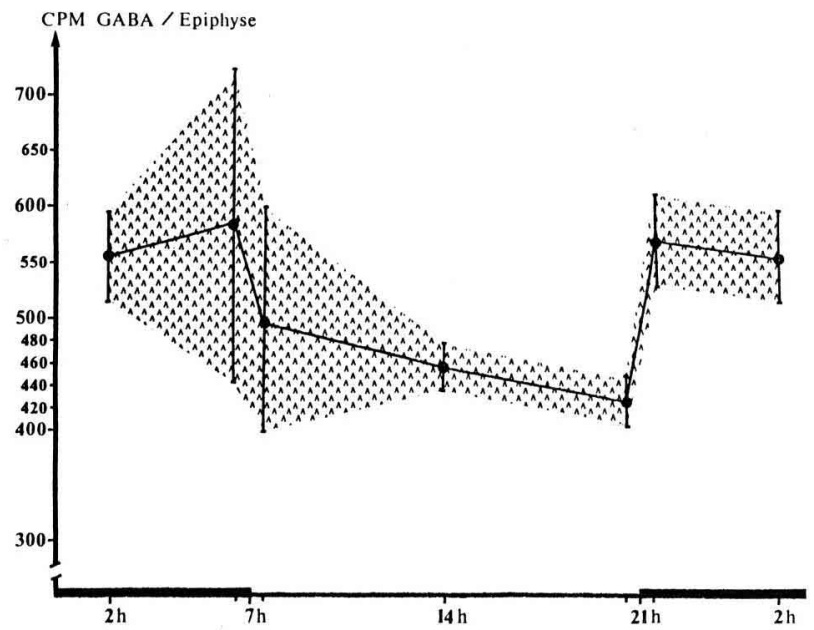

a) Photopériode longue

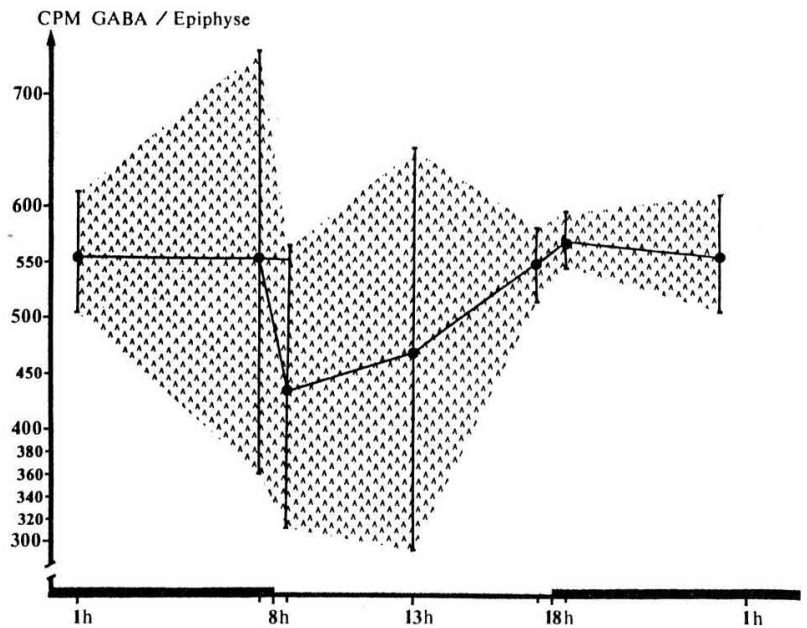

b) Photopériode courte

FIG. 1. - Etude du rythme circadien de l'incorporation du GABA tritié par l'épiphyse de souris: mesure de l'activité moyenne du ${ }^{3} H$ GABA par épiphyse.

a) En photopériode longue :

- La différence entre les moyennes ( $F$ de Fischer) est significative avec $p<0,001$.

- Comparaison des lots deux à deux ( $\Delta$ de Scheffe) : le lot 5 est différent du lot $6(p<0,001)$; le lot 2 est différent du lot $4(p<0,01)$; le lot 1 est différent du lot $4(p<0,05)$; le lot 1 est différent du lot $5(p<0,05)$; le lot 2 est différent du lot $5(p<0,01)$.

b) En photopériode courte le F de Fisher n'est pas significatif. 


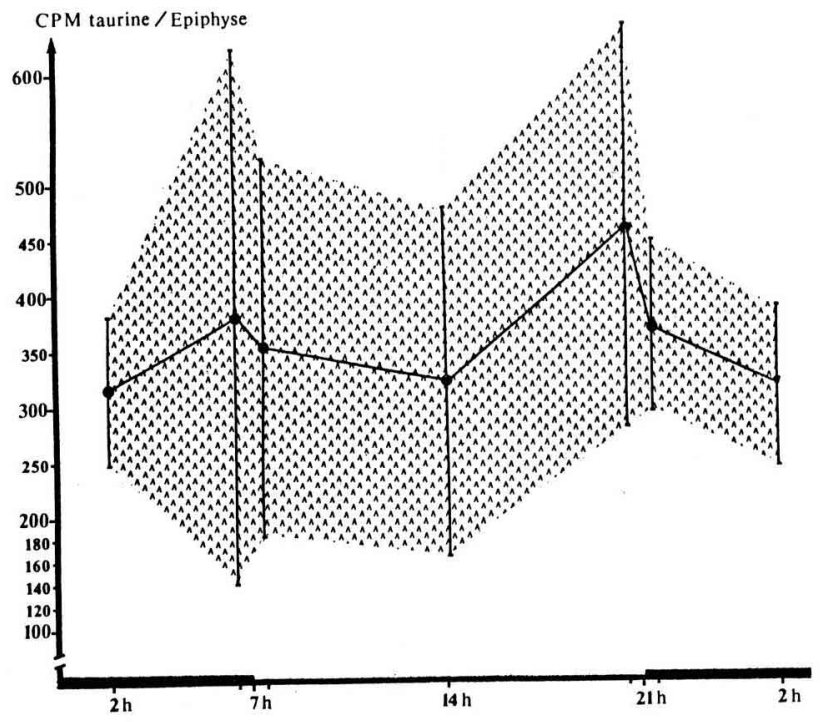

a) Photopériode longue

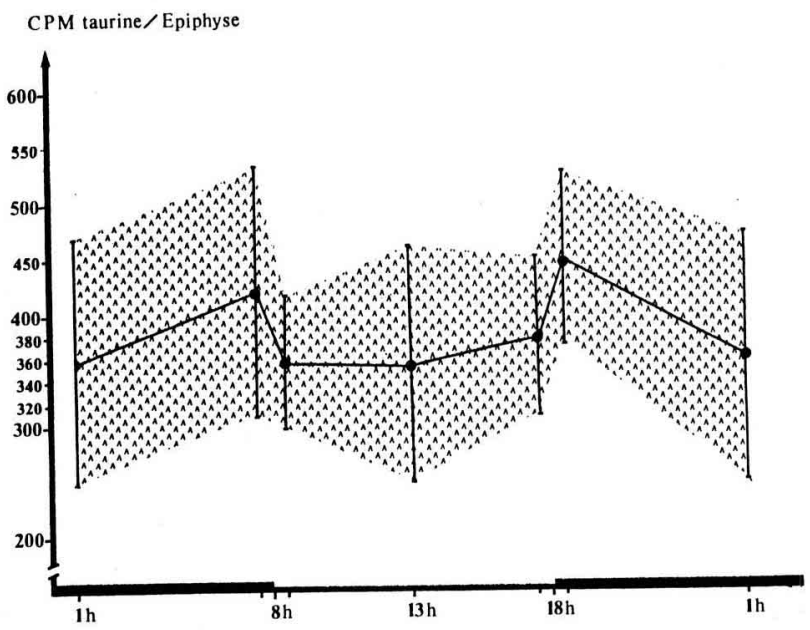

b) Photopériode courte

FIG. 2. - Etude du rythme circadien de l'incorporation de taurine tritiée par l'épiphyse de souris: mesures de l'activité moyenne de la ${ }^{3} \mathrm{H}$ Taurine par épiphyse.

a) En photopériode longue : $F$ de Fisher non significatif

b) En photopériode courte: $\mathrm{F}$ de Fisher non significatif. 


\section{Discussion.}

La constance des résultats obtenus après l'incorporation des deux traceurs au niveau du cervelet montre qu'il existe une homogénéité d'imprégnation dans les divers lots d'animaux. Les faibles quantités incorporées montrent par ailleurs que le captage du GABA et de la taurine par le cervelet est très atténué par la barrière hémato-encéphalique.

Au niveau de l'épiphyse les observations sont très différentes.

$A-G A B A$.

L'étude de l'incorporation du GABA tritié par l'épiphyse de souris permet de décrire un rythme nycthéméral de captage chez les animaux soumis à des photopériodes longues (14 h L/10 h Ob). L'avidité de la glande pour cet acide aminé est maximale au cours de la nuit et minimale dans la journée. Ces variations sont parallèles aux variations de l'activité épiphysaire, caractérisées par la décharge nocturne de NA et l'activation consécutive de la NAT et de l'hydroxyindole-0-méthyltransférase (HIOMT). II convient toutefois de noter que ces dernières données n'ont été jusqu'à présent vérifiées chez la souris que par Upson et al. en 1976 et par Matsushima et al. en 1977. Le captage de GABA tritié par la pinéale est important pendant la nuit, lorsque la glande est active, et faible le jour. Il est modifié par le changement d'éclairement et ce phénomène est particulièrement brutal lors du passage du jour à la nuit.

Chez les souris placées en photopériode courte, on constate une très grande variabilité de l'incorporation du GABA marqué dans les lots étudiés. Dans ces conditions, il n'est pas possible de décrire avec certitude une évolution du captage au cours du nycthémère. Cependant, les courbes obtenues semblent assez parallèles à celles qu'on observe avec les photopériodes longues.

Les différences observées entre les deux groupes semblent montrer que le rôle du GABA est nettement plus marqué lorsque l'épiphyse voit ralentir son activité par le passage d'un régime photopériodique neutre (12 h L/12 h Ob) à un régime lumineux plus long (14 h L/10 h Ob). Inversement, son effet est moins bien mis en évidence lorsque l'épiphyse est activée par le passage à un régime court $(10 \mathrm{~h}$ $\mathrm{L} / 14 \mathrm{~h} \mathrm{Ob})$.

Ces constatations s'expliquent mal dans l'état actuel des connaissances sur l'effet stimulant ou inhibiteur du GABA vis-à-vis de l'épiphyse de la souris.

Notre travail concernant le GABA exogène aboutit à des résultats strictement inverses de ceux de Waniewski et Suria (1977), qui décrivent chez le rat un rythme circadien du GABA endogène avec un maximum à $15 \mathrm{~h}$ et un minimum pendant la nuit ; il faut admettre que l'avidité de la glande pour le GABA circulant est en relation avec les périodes de synthèse alors que le GABA endogène s'accumule pendant les périodes de repos.

\section{$B-$ Taurine.}

L'incorporation épiphysaire de taurine tritiée chez la souris semble assez stable au cours du nycthémère, tant en photopériode longue que courte. Elle ne semble pas non plus sujette à des variations circadiennes. On peut penser que, 
contrairement au GABA, cette substance n'intervient pas uniquement comme médiateur de la régulation de la synthèse des indolamines dans l'épiphyse de la souris. Les grandes fluctuations individuelles et nycthémérales observées dans nos expériences semblent plutôt montrer que la taurine exogène est captée en fonction des besoins métaboliques de la glande, ce qui pourrait éventuellement masquer une activité neuromodulatrice.

\section{Conclusion.}

1) L'étude de l'incorporation du GABA tritié par l'épiphyse de la souris permet de décrire un rythme nycthéméral de captage chez les animaux soumis à des photopériodes longues ( $14 \mathrm{~h} \mathrm{L/10} \mathrm{h} \mathrm{Ob)}$.

L'avidité de la glande pour cet acide aminé marqué est maximale au cours de la nuit et minimale pendant la journée (fig. 1 et 2). Les cycles de captage du GABA tritié par l'épiphyse de la souris pendant le nycthémère semblent suivre le rythme d'activité de la glande.

L'augmentation brutale du captage au début de la scotophase nous est apparue comme une observation importante, de même que la diminution de l'incorporation au début de la période lumineuse, qui est cependant plus progressive. L'épiphyse est tout particulièrement sensible aux changements brusques de l'environnement lumineux.

Chez les souris placées en photopériode courte il n'est pas possible de décrire une évolution du captage au cours du nycthémère.

2) L'incorporation de taurine tritiée chez la souris semble assez stable au cours du nycthémère et ne paraît pas influencée par des modifications de la photopériode.

Reçu en mai 1987

Accepté en décembre 1987.

\section{Références}

ADLER R., 1983. Taurine uptake by chick embryo retinal neurones and glial cells in purified culture J. Neurosci. Res., 10, 369-379.

AXELROD J., ZATZ M., 1977. The $\beta$-adrenergic receptor and the regulation of circadian rhythms in the pineal gland, 249-268. In LITTWACK G., Biochemical actions of hormones, vol. IV, Acad. Press, New York.

BARBEAU A., TSUKADA Y., INONE N., 1968. Neuropharmacological and behavioural effects of taurine, 253-266. In HUXXABLE R., BARBEAU A., Taurine, Raven Press, New York.

BASKIN S. I., DAGIRMANJIAN R., 1973. The effects of taurine on the pigmentation of the bullfrog tadpole. Comp. Biochem. Physiol., 44A, 297-302.

EBADI M., CHAN A., 1980. Characteristics of GABA binding sites in bovine pineal gland. Brain Res. Bull., 5 (suppl. 2), 179-187.

EBELS I., BENSON B., LARSEN B. R., 1980. Biosynthesis of taurine by rat pineals in vitro. J. neur. Transm., 48, 101-117.

FORCHETTI C. A., 1979. Evidence for a tonic GABAergic control of serotonin neurons in the median raphe nucleus. Brain Res., 208, $181-186$. 
GROSSO D. S., BRESSLER B., BENSON B., 1978. Circadian rhythm and uptake of taurine by rat pineal gland. Life Sci, 22, 1789-1798.

KANAZAWA I., IVERSEN L. L., KELLY J. S., 1976. Glutamate decarboxylase activity in the rat posterior pituitary, pineal gland, dorsal root ganglion and superior cervical ganglion. $J$. Neurochem., 27, 1267-1270.

LABELLA F., VIVIAN S., OUEEN G., 1968. Abundance of cystathionine in the pineal body. Free amino acids and related compounds of bovine pineal, anterior and posterior pituitary and brain. Biochim. biophys. Acta, 158, 286-288.

LEGAIT H., OBOUSSIER H., 1977. Etude interspécifique des corrélations statistiques existant entre le volume et le poids de la glande pinéale et les poids encéphalique et somatique dans deux groupes de mammifères. Etude intraspécifique des corrélations entre glande pinéale et encéphale chez le rat et chez l'homme. Bull. Assoc. Anat., 61, 123-132.

MATA M. M., SCHRIER B. K., KLEIN D. C., WELLER J. L., CHIOU C. Y., 1976. On GABA function and physiology in the pineal gland. Brain Res., 118, 383-394.

MATSUSHIMA S., KACHI T., MUKAI S., MORISAWA Y., 1977. Functional relationships between sympathetic nerves and pinealocytes in the mouse pineal : quantitatives electronmicroscopic observations. Arch. histol. jap., 40 (suppl.), 279-291.

NAMINA M., OKAMOTO K., SAKAI Y., 1982. Taurine acts on presynaptic autoreceptor for GABA in the cerebellum : effects on $\mathrm{Ca}^{+}$influx and GABA release. Jap. J. Pharmacol., 32, 746-749.

ROSENBLUM I. Y., BENSON B., BRIA C. F., MCDONNELL D., HRUBY V. J., 1979. Localization and chemical characterization of a partially purified bovine pineal antigonadotropin. $J$. neur. Transm., 44, 197-220.

SCHON F., BEART P. M., CHAPMAN D., KELLY J. S., 1975. On GABA metabolism in the gliocyte Cells of the rat pineal gland. Brain Res., 85, 479-490.

UPSON R. H., BENSON B., SATTERFFIELD Y., 1976. Quantitation of ultrastructural changes in the mouse pineal in response to continuous illumination. Anat. Rec., 184, 311-324.

VELLAN E. J., GJESSING L. R., STALSBERG H., 1970. Free aminoacids in the pineal and pituitary glands of human brain. J. Neurochem., 17, 699-701.

WANIEWSKI R. A., SURIA A., 1977. Alterations in gamma-aminobutyric acid content in the rat superior cervical ganglion and pineal gland. Life Sci., 21, 1129-1142.

YOUNG J. A., BROWN D. A., KELLEY J. S., SCHON F., 1973. Autoradiographic localization of sites of ${ }^{3} \mathrm{H}$-GABA accumulation in peripheral autonomic ganglion. Brain Res., 63, 479-486. 